\title{
Comparación de la higiene oral en niños con y sin discapacidad: importancia de la supervisión
}

DOI: $10.46932 /$ sfjdv2n1-001

Received in: November 1st, 2020

Accepted in: December 30th, 2020

\author{
Beatriz Romero Triviño \\ Graduada en Odontología \\ Institución: Facultad de Odontología, Universidad Complutense de Madrid (UCM) \\ Avenida Ramon y Cajal S/N 28040 Madrid. Spain \\ bearom03@ucm.es \\ Margarita Iniesta Albentosa \\ Profesora asociada de la Facultad de Odontología UCM \\ Institución: Facultad de Odontología, Universidad Complutense de Madrid (UCM) \\ Avenida Ramon y Cajal S/N 28040 Madrid. Spain \\ margaini@ucm.es
}

\section{RESUMEN}

Objetivo: El objetivo de este estudio fue comparar la salud oral de niños plurideficientes con un grupo control sin discapacidad.

Material y método: se incluyeron en el estudio 50 sujetos ( 25 en el grupo test y 25 en el grupo control). Las variables analizadas fueron índice de placa (IP), índice de dientes con caries/ausentes/obturados (CAOD), tinción, maloclusión y empleo de aparatología.

Resultados: Con respecto a la media del IP y el CAOD, no se observaron diferencias estadísticamente significativas entre el grupo test y control $(\mathrm{p}=0,824$ y $\mathrm{p}=0,055$ respectivamente). La media de dientes ausentes y el porcentaje de tinción y de maloclusión fue mayor en el grupo test $(p=0,012, P=0,002, p$ $=0,001$, respectivamente); y el índice de restauración fue mayor en el grupo control $(\mathrm{p}=0,017)$. Conclusión: En este estudio, la prevalencia del IP y el CAOD no difiere entre ambos grupos, lo que podría significar que la supervisión del cepillado de los niños con plurideficiencia, por parte de sus cuidadores, repercute de alguna manera en su salud oral equiparándola a la de la población sin discapacidad.

Palabras-clave: higiene oral, salud oral, discapacidad, niños

\section{INTRODUCCIÓN}

La plurideficiencia o polidiscapacidad se define como la disfunción severa o profunda de dos o más áreas del desarrollo, incluyendo siempre el déficit cognitivo. Se manifiesta a través de un gran retraso del desarrollo psicomotriz, acompañado frecuentemente de déficits sensoriales (visión, audición...), crisis epilépticas y problemas de salud: digestivos, respiratorios, cutáneos, dentales, cardiovasculares... (BOUKERAS, 2008) 
Según el Instituto Nacional de Estadística (INE), en 2008 había en España 3.847.900 personas con discapacidad, lo que representa un $8,5 \%$ de la población, de la cual un 11,29\% se encuentra en la Comunidad de Madrid. (INE, 2008)

Actualmente, el número de personas con pluridiscapacidad se sitúa en un 0,5-0,7\% a nivel mundial según la OMS (WHO, 2011).

Los problemas comunes bucales, tales como caries o enfermedades de las encías, afectan a todos los niños, pero lo niños con discapacidades y otras necesidades especiales poseen una mayor prevalencia de enfermedades (SILVESTRE, 2004). Algunos de los factores que contribuyen a una salud bucal deficiente en los niños con discapacidades son las condiciones bucales genéticas, limitaciones físicas, dificultad para la remoción de placa, flujo salival reducido, medicamentos y dietas restringidas (RAHMAN, 2007).

Algunos autores (WARD, 2019; PETROVIC, 2016) indican que la salud bucal de los pacientes con algún tipo de discapacidad es significativamente peor que la de la población general, ya que, existe una prevalencia universal de enfermedad periodontal y una higiene bucal más pobre.

La presencia de un cuidador prestando atención a la salud oral y a los hábitos de higiene en la población discapacitada debería tener un impacto sobre su salud oral, por lo tanto, en el presente estudio, sobre el estado de salud oral en un grupo reducido de niños plurideficientes de la Comunidad de Madrid, se plantea como objetivo general, comparar los problemas bucodentales entre niños plurideficientes supervisados y niños sanos.

Además, como objetivos específicos:

- Comparar el índice CAO entre niños sanos y plurideficientes

- Comparar el índice de placa entre ambos grupos

- Comparar los porcentajes de maloclusión y empleo de aparatología

- Comparar el porcentaje de tinciones

\section{MATERIAL Y MÉTODO}

\subsection{DISEÑO DEL ESTUDIO}

Se trata de un estudio analítico transversal, en el que se estudia la prevalencia de caries, índice de placa y otras variables de salud oral en una población de la Comunidad de Madrid plurideficiente y su comparación con un grupo control de pacientes sin minusvalías (WHO, 2013) 


\subsection{SELECCIÓN DE LA MUESTRA}

Para la selección de la muestra se emplea un muestreo no probabilístico de casos consecutivos. Se seleccionaron 25 sujetos procedentes de un colegio de pacientes especiales de Getafe (grupo test), y 25 sujetos de entre los que acudían a la Clínica de la Facultad de Odontología de UCM (Universidad Complutense de Madrid) (grupo control). El colegio escogido es un centro específico de educación especial concertado con la Comunidad de Madrid de ámbito local y autonómico, que forma parte de un grupo de empresas de acción social, con un compromiso ético en pro de la persona con discapacidad intelectual y sus familias, actuando a través de la educación como medio de normalización en todos los ámbitos de su vida.

Se establecieron una serie de criterios de inclusión y exclusión en el grupo test:

Criterios de inclusión:

- $\quad$ Edad $\leq$ de 20 años

- Sujetos que están bajo la supervisión de educadores especiales

- Sujetos con el suficiente nivel cognitivo como para desarrollar una interacción con el personal de referencia

Criterios de exclusión: sujetos con enfermedades que conllevan asociada una limitación psicomotora incapacitante como parálisis cerebral, espina bífida u otras lesiones

El grupo control se seleccionó emparejando por sexo y edad con el grupo test.

\subsection{VISITAS DEL ESTUDIO}

Visita de selección. Todos los sujetos potenciales fueron evaluados con respecto a los criterios de inclusión y exclusión

Visita del estudio. A los pacientes seleccionados se les realizó un registro de todas las variables clínicas detalladas a continuación.

\subsection{RECOGIDA DE DATOS}

Examinadores. La recogida de datos fue llevada a cabo por alumnos de la Facultad de Odontología de la UCM. La exploración de las variables se realizó mediante inspección visual con luz natural y espejo de exploración. 
Variables demográficas. Se recogieron datos como la edad, sexo y otras variables psicomotoras como lenguaje limitado y movilidad reducida.

Variables clínicas. Se incluyeron todos los dientes en el examen, excepto los terceros molares. Las variables analizadas fueron:

- Índice de placa: análisis dicotómico basado en la presencia/ausencia de placa detectada con una tinción de eritrosina (Plac Control $® ;$ Dentaid, Barcelona, España). Se evaluaron 4 localizaciones por diente, 3 por vestibular y 1 por lingual/palatino (LÖE, 1967).

- Índice CAO: Se exploraron 5 superficies por diente valorando la existencia de caries (C), ausencias (A) u obturaciones (O) en dientes permanentes (KLEIN, 1938). En dientes temporales se recogió el índice co de la misma forma (para dientes con caries u obturaciones). En dentición mixta se calculó el CAO + co. A efectos prácticos, como la mayoría de los sujetos presentaba una dentición permanente, muy pocos dentición mixta y ninguno dentición temporal, aunamos la nomenclatura de estos índices bajo el nombre general de índice CAO

- Índice de restauración (IR) que se calcula como (O/CAO) x100 e informa sobre la atención odontológica restauradora recibida y estima la dimensión de ésta acción sobre el conjunto de la enfermedad de caries (GERENCIA ASISTENCIAL MADRID, 2017).

- Tinción: Se valoró la presencia /ausencia de superficies dentales con alteración del color original excluyendo manchas blancas de descalcificación

- Maloclusiones: Se evaluó la presencia/ausencia de maloclusión, tomando como referencia la posición de molares y caninos, valorando el plano transversal, anteroposterior y vertical (ANGLE, 1899).

- Aparatología dentaria: Se evaluó la presencia/ausencia de prótesis u ortodoncia.

\subsection{ANÁLISIS DE LOS DATOS}

Para analizar la normalidad de la distribución de los datos se usó el test de Shapiro-Wilk. Para el análisis de las variables continuas se utilizó el test t de Student para muestras independientes, y la prueba chi cuadrado para los datos categóricos. Todos los datos se analizaron con el software IBM®, SPSS ${ }^{\circ}$, Statistics 20 (IBM Corporation, Armonk, Nueva York, EEUU) usando dos colas y un nivel de significación de $p<0,05$. 


\section{RESULTADOS}

\section{Descripción de la muestra}

\section{Grupo test}

La edad media (desviación estándar) de los pacientes en el grupo test fue de 13,96 (3,42), teniendo el más joven 7 años y el mayor 19 años. En cuanto a la distribución por sexos, encontramos 10 mujeres, con una edad media de 14,5 años (3,06); y 15 hombres con una edad media de 13,60 $(3,69)$ años. (tabla 1)

Tabla 1. Descripción de la población y comparación entre grupos en relación a las variables demográficas

\begin{tabular}{|c|c|c|c|c|c|}
\cline { 3 - 6 } \multicolumn{2}{c|}{} & $\begin{array}{c}\text { Grupo test } \\
(\mathrm{n}=25)\end{array}$ & $\begin{array}{c}\text { Grupo control } \\
(\mathrm{n}=25)\end{array}$ & Total $(\mathrm{n}=50)$ & $p$ \\
\hline \multirow{2}{*}{ Edad: media (DE) } & Total & $13,96(3,42)$ & $14,04(3,57)$ & $14(3,46)$ & 0,197 \\
\cline { 2 - 6 } & Hombres & $13,60(3,69)$ & $13,83(3,21)$ & $13,70(3,42)$ & 0,233 \\
\cline { 2 - 6 } & Mujeres & $14,50(3,06)$ & $14,23(4,00)$ & $14,35(3,55)$ & 0,862 \\
\hline Sexo: n (\%) & Hombres & $15(60)$ & $12(48)$ & $27(54)$ & 0,394 \\
\cline { 2 - 6 } & Mujeres & $10(40)$ & $13(52)$ & $23(46)$ & \\
\hline
\end{tabular}

n=número de sujetos, $\mathrm{DE}=$ desviación estándar

Del número total de pacientes, 7 (28\%) sujetos presentaban lenguaje limitado lo que restringía la comunicación, pero no la impedía; y 5 pacientes (20\%) presentaban movilidad limitada en alguna extremidad. (tabla 2)

Tabla 2. Descripción de la población según variables psicomotoras

\begin{tabular}{|c|c|c|c|c|}
\cline { 2 - 5 } \multicolumn{1}{c|}{} & Grupo test $(\mathrm{n}=25)$ & $\begin{array}{c}\text { Grupo control } \\
(\mathrm{n}=25)\end{array}$ & Total $(\mathrm{n}=50)$ & $p$ \\
\hline Lenguaje limitado: $\mathrm{n}(\%)$ & $7(28)$ & $0(0)$ & $7(14)$ & 0,004 \\
\hline Movilidad reducida: $\mathrm{n}(\%)$ & $5(20)$ & $0(0)$ & $5(10)$ & 0,018 \\
\hline
\end{tabular}

n: número de sujetos

\section{Grupo control}

La edad media de los pacientes en el grupo control era de 14,04 $(3,57)$, teniendo el más joven 7 años y el más mayor 20 años. En cuanto a la distribución por sexos, 13 fueron mujeres con una edad media de 14,23 (4,00) y 12 varones con una edad media de13,83 (3,21) (tabla 1) 
No se encontraron diferencias estadísticamente significativas en relación con la edad o el sexo entre grupos ( $p>0,05)$, lo que indica que los grupos estaban debidamente pareados en cuanto a estas variables (tabla 1 ).

Por las características psicomotoras de uno y otro grupo, encontramos diferencias estadísticamente significativas en cuanto a la variable lenguaje limitado y movilidad reducida entre grupos $(\mathrm{p}<0,05)($ tabla 2$)$

\section{Variables clínicas}

A la hora de realizar el examen intraoral, a 2 pacientes del grupo test no se les pudo completar la exploración porque fue imposible obtener su colaboración.

\section{Índice de placa (tabla 3):}

En el grupo test se observó una media (desviación estándar) del índice de placa de $51(31,9)$ y en el grupo control se observó una media del índice de placa de 41,70 (27,53).

No se observaron diferencias estadísticamente significativas entre ambos grupos en cuanto al índice de placa $(\mathrm{p}=0,284)$

Tabla 3. Comparación de la media(DE) del índice de placa entre el grupo test y el grupo control

\begin{tabular}{|c|c|c|c|c|c|c|}
\hline & $\begin{array}{c}\text { Grupo test } \\
(\mathrm{n}=23)\end{array}$ & $\begin{array}{c}\text { Grupo } \\
\text { control } \\
(\mathrm{n}=25)\end{array}$ & $\begin{array}{c}\text { Diferencia de } \\
\text { medias }\end{array}$ & $\begin{array}{c}\text { IC } \\
\text { Límite } \\
\text { inferior }\end{array}$ & $\begin{array}{c}\text { Límite } \\
\text { superior }\end{array}$ & $p$ \\
\hline $\begin{array}{c}\text { Índice de placa: } \\
\text { media (DE) }\end{array}$ & $51(31,9)$ & $41,7(27,53)$ & 9,29 & $-26,57$ & 7,97 & 0,284 \\
$\mathrm{n}=$ Tamaño muestral. DE= Desviación estándar. IC= Intervalo de confianza
\end{tabular}

Índice de caries (tabla 4):

En el grupo test se observó una media de índice de CAO de 3,52 $(3,39)$ presentando una media de caries de 1,52 (2,25), una media de dientes ausentes de 1,26(2,22) y una media de dientes obturados de 0,74(1,48). Además, este grupo presentaba una media de índice de restauración de 17,24(36,82). De las caries citadas anteriormente, el $85,72 \%$ se encuentra en dientes permanentes frente al $14,28 \%$ de caries en dientes temporales.

En el grupo control, se observó una media de índice de CAO de 1,88 (2,31) presentando una media de caries de $0,76(1,71)$ y una media de dientes obturados de $1,12(1,26)$. Estos sujetos presentaban una media de índice de restauración de 46,73 $(45,50)$ y no presentaban ninguna ausencia dentaria. 
Se encontraron diferencias estadísticamente significativas en el número de dientes ausentes $(\mathrm{p}=0,012)$ y en el IR ( $p=0,017)$ entre ambos grupos, pero no se observaron diferencias estadísticamente significativas ni en el número de dientes con caries $(\mathrm{p}=0,198)$, ni obturados $(\mathrm{p}=0,346)$, ni en la media del índice de CAO ( $\mathrm{p}=0,055)$, aunque este índice tendía a la significación. (tabla 4)

Tabla 4. Comparación de la media (desviación estándar) del número de dientes con caries, ausente, obturados, índice de CAO e índice de restauración entre el grupo test y el grupo control

\begin{tabular}{|c|c|c|c|c|c|c|}
\hline & $\begin{array}{c}\text { Grupo test } \\
(\mathrm{n}=23)\end{array}$ & $\begin{array}{c}\text { Grupo control } \\
(\mathrm{n}=25)\end{array}$ & $\begin{array}{c}\text { Diferencia } \\
\text { de medias }\end{array}$ & \multicolumn{2}{|c|}{$\begin{array}{c}\text { IC } \\
\text { Límite } \\
\text { inferior }\end{array}$} & \multicolumn{2}{c|}{$\begin{array}{c}\text { Límite superior } \\
\text { (DE) }\end{array}$} & $1,52(2,25)$ & $0,76(1,71)$ & 0,76 & $-1,93$ & 0,41 & 0,198 \\
\hline $\begin{array}{c}\text { Caries: media } \\
(\mathrm{DE})\end{array}$ & $0(0)$ & 1,26 & $-2,22$ & $-0,30$ & 0,012 \\
\hline $\begin{array}{c}\text { Ausentes: } \\
\text { media (DE) }\end{array}$ & $1,26(2,22)$ & $1,12(1,26)$ & $-0,38$ & $-0,42$ & 1,18 & 0,346 \\
\hline $\begin{array}{c}\text { Obturados: } \\
\text { media (DE) }\end{array}$ & $0,74(1,48)$ & $1,88(2,31)$ & 1,64 & $-3,31$ & 0,03 & 0,055 \\
\hline $\begin{array}{c}\text { CAO: media } \\
\text { (DE) }\end{array}$ & $3,52(3,39)$ & $46,73(45,50)$ & $-28,48$ & 5,50 & 53,46 & 0,017 \\
\hline $\begin{array}{c}\text { Índice } \\
\text { restauración: } \\
\text { media (DE) }\end{array}$ & $17,24(36,82)$ & & & & & \\
\hline
\end{tabular}

n= Tamaño muestral. DE= Desviación estándar. $\mathrm{IC}=$ Intervalo de confianza

\section{Tinción (tabla 5):}

El 30,4\% de los pacientes plurideficientes (7 sujetos) presentaban tinciones en el esmalte sobre todo a nivel del grupo anterior por vestibular. Observamos una diferencia estadísticamente significativa entre el grupo test y control $(\mathrm{p}=0,002)$, ya que, ninguno de los pacientes del grupo control presentaba tinciones.

\section{Aparatología (tabla 5):}

Ninguno de los pacientes discapacitados usaba prótesis ni aparatos ortodóncicos. En cambio, en el grupo control se observó que 2 pacientes se encontraban en tratamiento de ortodoncia, por lo que, encontramos una diferencia estadísticamente significativa entre ambos grupos $(\mathrm{p}=0,165)$

\section{Maloclusión (tabla 5):}

En el grupo test, el 73,9\% de los pacientes (17 sujetos) presentaba algún tipo de maloclusión, mientras que en el grupo control, sólo el 28\% de los pacientes ( 7 sujetos) presentaba algún tipo de maloclusión. Se observó una diferencia estadísticamente significativa entre ambos grupos en cuanto a la presencia de maloclusión ( $\mathrm{p}=0,001)$. 
En cuanto al tipo de maloclusión, tal y como se expone en la tabla 6, la mordida cruzada es la maloclusión con mayor prevalencia en un total de 7 sujetos $(14,58 \%$ del total).

Tabla 5. Comparación del número de sujetos y porcentaje entre grupo test y control en relación a las variables tinción, maloclusión y aparatología

\begin{tabular}{|c|c|c|c|c|}
\hline & Grupo test $(\mathrm{n}=23)$ & $\begin{array}{c}\text { Grupo control } \\
(\mathrm{n}=25)\end{array}$ & Total $(\mathrm{n}=48)$ & $p$ \\
\hline Tinción: $\mathrm{n}(\%)$ & $7(30,4)$ & $0(0)$ & $7(14,6)$ & 0,002 \\
\hline Maloclusión: $\mathrm{n}(\%)$ & $17(73,9)$ & $7(28)$ & $24(50)$ & 0,001 \\
\hline Aparatología: $\mathrm{n}(\%)$ & $0(0)$ & $2(8)$ & $2(4,16)$ & 0,165 \\
\hline
\end{tabular}

$\mathrm{n}=$ número de sujetos

Tabla 6. Comparación del tipo de maloclusión dentaria entre grupo test y control

\begin{tabular}{|c|c|c|c|c|}
\hline & $\begin{array}{c}\text { Grupo test } \\
(\mathrm{n}=23)\end{array}$ & $\begin{array}{c}\text { Grupo control } \\
(\mathrm{n}=25)\end{array}$ & $\begin{array}{c}\text { Total } \\
(\mathrm{n}=48)\end{array}$ & $p$ \\
\hline Mordida cruzada: n (\%) & $6(26,08)$ & $1(4)$ & $7(14,58)$ & 0,044 \\
\hline Mordida abierta: n (\%) & $3(13,04)$ & $1(4)$ & $4(8,33)$ & 0,338 \\
\hline Apiñamiento: n (\%) & $2(8,7)$ & $4(16)$ & $6(12,5)$ & 0,668 \\
\hline Clase III: n (\%) & $2(8,7)$ & $1(4)$ & $3(6,25)$ & 0,601 \\
\hline Clase II: n $(\%)$ & $2(8,7)$ & $0(0)$ & $2(4,16)$ & 0,224 \\
\hline
\end{tabular}

$\mathrm{n}=$ número de sujetos

\section{DISCUSIÓN}

Se han realizado estudios (BLANCO, 2016; PINI, 2016) que evalúan la higiene oral tanto en niños discapacitados como sin discapacidad, y a la hora de compararlo con nuestros datos, se observan índices de placa muy similares de 55,3\% y $35 \%$ respectivamente. Esto demuestra que nuestros pacientes se encuentran en la media respecto al índice de placa. Zhou et al., (2019) observaron la relación entre el mayor índice de placa en niños con discapacidad con el peor funcionamiento intelectual, la deficiencia en habilidades prácticas y la corta duración del cepillado dental.

Numerosos autores, (THIKKURISSY, 2009; HIDAS, 2010; JAWADI, 2004) han observado como aquellas enfermedades que impiden una alimentación normal y que dependen de la alimentación por sonda de gastrostomía producen una mayor acumulación de cálculo y unos valores mas elevados de índice gingival, debido en su mayoría a la falta de aclaramiento de la cavidad oral.

En la última encuesta de salud oral de la Comunidad de Madrid (GERENCIA ASISTENCIAL MADRID, 2017) se recogen datos sobre niños de 6 a 15 años. En cuanto a los dientes con caries, se observa una media de 0,47 en ausencias dentarias se observan una media de 0,01 y en dientes con obturaciones una media de 0,445. Estos datos, son ligeramente inferiores a los recogidos en el grupo 
control de nuestro estudio, probablemente debido a la variabilidad demográfica y al tamaño muestral; por lo tanto, nuestros datos acerca del grupo control se encuentran por encima de la media.

El riesgo de caries dental está íntimamente relacionado con la presencia de hipoplasia de esmalte, el mal estado nutricional y los medicamentos que reducen la saliva o contienen azúcar. Ciertas enfermedades que cursan con disfagia oral o reflujo producen un embolsamiento de alimentos y líquidos, promoviendo un aumento de caries dental y la posibilidad de exponer a los dientes a contenido gástrico ácido. (KENNETH, 2013)

En el estudio de Cabrita JP et al., (2017) se estudió la prevalencia de maloclusiones comparándolo entre ambos grupos. Ellos observaron que tan sólo el $25 \%$ de los pacientes con discapacidad no presentaban maloclusión, tal y como se observa en nuestro estudio, sin embargo, en su estudio la clase II de Angle tenía la mayor prevalencia. La falta de experiencias orales motoras y musculares, da lugar a una deficiencia en el desarrollo normal esquelético de los niños con discapacidad provocando con ello maloclusiones dentales y esqueléticas. (KENNETH, 2013)

Los valores similares en cuanto al índice de placa entre el grupo con y sin discapacidad probablemente se deba en su mayoría al empleo de un cuidador. Esto se demuestra en el estudio de Costa et al., (2016), en el que se estudiaban pacientes con diferentes grados de discapacidad y se observaba que aquellos con enfermedades más severas, poseían mejor higiene oral debido a la supervisión de un cuidador. Shin y Saeed (2013), por su parte, observaron como la higiene oral en este tipo de pacientes mejoraba con las instrucciones y que las áreas con peor higiene oral eran aquellas que precisaban una mayor compresión y destreza de las instrucciones como el cepillado de los dientes anteriores.

En la comunidad de Madrid se presta atención bucodental a todos los pacientes de 6 a 18 años con discapacidad física o psíquica reconocida que les impida la colaboración en un gabinete tradicional. Para proporcionar las mayores garantías de seguridad en estos pacientes, estos tratamientos se realizan en la Unidad de Salud Bucodental ubicada en el Hospital Infantil Universitario Niño Jesús. A esta unidad se derivan los usuarios que a causa de su déficit no son capaces de mantener el autocontrol necesario que permita una adecuada atención sin tratamientos sedativos. El tratamiento se lleva a cabo con anestesia general y en quirófano en un medio hospitalario (PORTAL SALUD MADRID, 2020).

Se encontraron una serie de limitaciones durante la realización del estudio. El hecho de que fueran varios observadores los que decidieran las categorías de las variables de estudio puede originar cierta variabilidad diagnóstica intrínseca al proceso de diagnóstico clínico. Además, el empleo de luz 
solar, y la imposibilidad de exploración de determinados pacientes, provoca una gran dificultad diagnóstica.

\section{CONCLUSIONES}

El empleo de un cuidador supervisando la higiene oral de los pacientes discapacitados, permite una mejora en la eliminación de placa en este tipo de paciente, lo que se demuestra al observar diferencias estadísticamente significativas frente a pacientes sin discapacidad en relación al índice de placa, índice CAOD, caries y dientes obturados. Aun así, aquellas variables dependientes de la enfermedad y la medicación tales como la tinción, maloclusiones o dientes ausentes sí que presentan diferencias estadísticamente significativas entre ambos grupos.

\section{AGRADECIMIENTOS}

Me gustaría agradecer al grupo de alumnos del APs y de la UCM por la recogida de datos para el estudio, así como por su labor asistencial en la prevención oral y ayuda a los pacientes plurideficientes de diferentes centros de pacientes especiales de Madrid. 


\section{BIBLIOGRAFÍA}

ANGLE, E. (1899) Classification of Malocclusion. Dental Cosmos. 74 (248-264); 350-357

BLANCO, M; PÉREZ-RÍOS, M; SANTIAGO-PÉREZ, M.I; SMYTH, E. (2016 Oct) Oral health and higiene status in galician schoolchildren. 85(4):204-209.

BOUKERAS, A. (2008) Évolution cognitive et socio-émotionnelle d’enfants ply-handicapés béneficiant de soins intensifs institutionnels. Bruxelles: De Boeck; En J.L. Adrien, BECS: Batterie d'evaluation cognitive et socio-émotionnelle p. 233-256.

CABRITA, J.P; BIZARRA, M.F; GRAÇA, S.R. (2017) Prevalence of malocclusion in individuals with and without intellectual disability: A comparative study. Special Care Dentistry. 37(4):181-186.

COSTA, A.A.; DELLA BONA, Á.; TRENTIN M.S. (2016 Jan-Feb). Influence of Different Intellectual Disability Levels on Caries and Periodontal Disease. Brazilian Dental Journal. 27(1):52-5.

GERENCIA ASISTENCIAL DE ATENCIÓN PRIMARIA DE MADRID. (2017) Estado de salud bucodental en la Comunidad de Madrid 2015/2016. 3 ed, España.

HIDAS, A; COHEN, J; BEERI, M; SHAPIRA, J; STEINBERG, D; MOSKOVITZ, M. (2010) Salivary bacteria and oral health status in children with disabilities fed through gastrostomy. Int $\mathrm{J}$ Paediatr Dent. 20(3):179-185

INE. (2008) Encuesta de Discapacidad, autonomía personal y situaciones de Dependencia (EDAD) [Internet]. Instituto Nacional de Estadística 2008. Disponible en: https://www.ine.es/prensa/np524.pdf

JAWADI, A.H; CASAMASSIMO, P.S; GRIFFEN, A; ENRILE, B; MARCONE, M. (2004) Comparison of oral findings in special needs children with and without gastrostomy. Pediatr Dent. 26 (3):283-288

KENNETH, W; NORWOOD, REBECCA L; SLAYTON, D.D.S. (2013 March) Oral Health Care for Children With Developmental Disabilities. Pediatrics.131(3):614-619

KLEIN, H; PALMER, C.E; KNUTSON, J.W. (1938) Studies on dental caries. Dental status and Dental Needs of elementary school children. Public Health Report. 53:751-765

LÖE, H. (1967) The Gingival Index, the Plaque Index, and the retención Index. J Periodontol. 38: 610616.

PETROVIC, B.B; PERIC, T.O; MARKOVIC, D..LJ; BAJKIN, B.B; PETROVIC, D; BLAGOJEVIC, D.B; VUJKOV, S. (2016 Dec) Unmet oral health needs among persons with intellectual disability. Res Dev Disabil. 59:370-377

PINI, D.M; FRÖHLICH, P.C; RIGO, L. (2016 Dec) Oral health evaluation in special needs individuals. Einstein (Sao Paulo) 14(4):501-507.

PORTAL DE SALUD MADRID. (2020) Programa de atención dental infanto/juvenil (PADI) (6 a 16 años). [Internet]

Disponible en: http://www.madrid.org/cs/Satellite?cid=1354552161373\&language=es\&pagename=PortalSalud\%2FPag e\%2FPTSA_pintarContenidoFinal\&vest=1354552161373 
RAHMAN, A; ZAMANI, M.P.H. (2007) Notas sobre salud y seguridad (California Childcare Health Program). Salud bucal para niños con discapacidades y necesidades especiales. (rev. 7/07)

SHIN, C.J; SAEED, S. (2013) Toothbrushing barriers for people with developmental disabilities: a pilot study. Spec Care Dentist. 33(6):269-74

SILVESTRE, F.J. (2004) Odontología en pacientes discapacitados. Valencia: Laboratorios Kin. THIKKURISSY, S; LAL, S. (2009) Oral health burden in children with systemic diseases. Dent Clin North Am. 53(2):351-357

WARD, L. M., COOPER, S. A., HUGHES-MCCORMACK, L., MACPHERSON, L., AND KINNEAR, D. (2019) Oral health of adults with intellectual disabilities: a systematic review. Journal of Intellectual Disability Research. 63: 1359- 1378

WHO. (2011) Informe mundial sobre la discapacidad [internet]. OMS 2011. Disponible en: http://www.who.int/disabilities/world_report/2011/summary_es.pdf

WHO. WORLD HEALTH ORGANIZATION. (2013) Oral Health Surveys Basic Methods. 5 ed. Geneva: WHO

ZHOU, N; WONG, H.M; MCGRATH, C. (2019) Oral health and associated factors among preschool children with special healthcare needs. Oral Dis. 25: 1221- 1228. 